\title{
Traces, réincarnations et durées longues dans les Amériques
}

\author{
Patrick Imbert ${ }^{1}$
}

\begin{abstract}
Résumé: On explore d'abord les divers types de durées longues qui permettent d'affirmer des identités stables dans les nations nouvelles des Amériques afin de déterminer qui définit la nation et qui, en corollaire, se situe à ses marges. Ensuite, on analyse la question de la réincarnation dans les romans contemporains comme moyen d'établir une durée longue en fonction d'une relecture du canon historique et de la reconnaissance de tous et des exclusions que, désormais, on critique. Finalement, on ouvre sur les diverses facettes des multiculturalismes tels qu'ils s'expriment dans les Amériques.
\end{abstract}

Mots clés : Amériques, histoire, identité, réincarnations, multiculturalisme, trace

Resumo: Explora-se primeiramente diversos tipos de duração longa que permitem afirmar identidades estáveis nas nações novas das Américas a fim de determinar quem define a nação e quem, em consequência, se situa à margem. Logo após, analisa-se a questão da reencarnação em romances contemporâneos como meio de estabelecer a longa duração em função de uma releitura do cânone histórico e do reconhecimento de todos e das exclusões as quais passamos a criticar. Finalmente, abre-se sobre as diversas faces dos multiculturalismos tais como eles se exprimem nas Américas.

Palavras-chave: Américas, história, identidade, reencarnações, multiculturalismo, vestígio

«Mon véritable pays est là où je deviens ce que je veux être» ${ }^{2}$

\section{La durée longue}

Pour Gérard Bouchard ${ }^{3}$, la durée longue est ce que les collectivités neuves du Nouveau-Monde ne possèdent pas par rapport à l'Europe et c'est ce qu'elles cherchent à mettre en place. Cette durée longue peut s'établir en fonction de différentes perspectives :

1 Patrick Imbert (1948) est professeur titulaire à l'Université d'Ottawa, titulaire de la Chaire de Recherche de l'Université: « Canada : Enjeux sociaux et culturels dans une société du savoir », (www.canada.uottawa.ca/ enjeux) et Président de l'Académie des arts et des sciences humaines de la Société royale du Canada. Il a publié 25 livres concernant les Amériques, l'analyse du discours et la littérature québécoise.

2 Ying Chen, Quatre mille marches, 2004, p. 12.

3 Gérard Bouchard, Genèse des nations et cultures du Nouveau Monde, Montréal, Boréal, 2001. 
1/Celle de Sarmiento ${ }^{4}$ et sa volonté de faire dominer les valeurs de l'Europe qu'il ne faut pas quitter, ce qui a pour conséquence le découpage continental entre barbarie/ civilisation, l'intérieur autochtone et créolisé étant la barbarie tandis que les ports ouverts sur l'Europe sont la civilisation. Cela conduit à une forme de structure de dominance coloniale, à l'exclusion et même au génocide des communautés indigènes établies depuis très longtemps ainsi que le renforcement du mythe des lumières et du progrès universel et homogénéisant tandis que l'autre, irrationnel et barbare, ne peut être intégré et est repoussé dans les marges ou éliminé. Autrement dit, la durée longue du mythe du progrès et son vecteur linéaire recontextualisant le narratif biblique du linéaire comme quête du jardin d'Éden perdu, en l'orientant de l'origine à une finalité tournée vers l'avenir, est moteur d'exclusion. Voilà qui est souligné par Tomson Highway, l'auteur autochtone canadien qui, dans Comparing Mythologies ${ }^{5}$, analyse le choc des mythes en valorisant celui des autochtones reposant sur le cercle, lui-même reposant sur la durée longue par le biais de l'autochtonie fondée sur la terre mère et non sur le récit adamique du mâle issu de la terre par la grâce d'un Dieu mâle. Il n'en reste pas moins que ces deux autochtonies, la chrétienne et l'indigène valorisent la durée longue.

2/Cette durée longue autochtone est reprise comme centralité de la culture au Mexique qui valorise le métissage monopolisé par l'État national. Il affirme que tous les Mexicains sont métissés et donc liés à des milliers d'années d'histoire précolombienne, ce qui a toutefois pour conséquence l'incapacité à reconnaître les communautés indigènes non-métissées, donc hors du progrès et de l'évolution historique définie par l'État et sa bureaucratie appuyée sur des artistes comme Diego Rivera qui, en des murales immenses, diffusent l'image de cette durée longue joignant l'antique et le nouveau mais dans une dynamique qui est contrôlée discursivement par l'État. On est donc loin d'Édouard Glissant ${ }^{6}$ qui parle de créolisation définie comme le métissage plus l'imprévisibilité. Dans son cas, on échappe à la linéarité de la narrativité institutionnelle pour ouvrir sur l'étude des traces et sur leur combinatoire aléatoire.

3/Le Canada français, quant à lui, en tant que société conquise par l'Angleterre, ne peut se prévaloir d'une capacité institutionnelle forte à s'affirmer dans une filiation qui lui donnerait la capacité de s'imposer. Ainsi le contrôle de la différence entre ser et estar dans la comparaison entre estar aqui et ser alguien lui échappe. Le ser alguien, l'affirmation identitaire forte arc-boutée sur le canon historique n'est pas au rendez-vous. Le grand récit de légitimation du lien avec les civilisations millénaires n'apparait pas car les autochtones du nord sont des nomades sans monuments ni codex. Sa combinaison avec les grands récits des indépendances et leur incarnation dans les monuments comme en Europe ou, à tout le moins, dans les statues équestres des libertadors des républiques des Amériques du Sud lui échappe aussi complètement. Il lui reste alors soit à ignorer l'américanité en se liant à l'Europe comme c'est le cas pour les penseurs « rouges » ou libéraux qui au nord vivent une dichotomie similaire au thème barbarie/civilisation de Sarmiento ${ }^{7}$, soit à rejeter l'Europe barbare, révolutionnaire, laïque et guerrière comme on le voit dans la conclusion de La terre paternelle de Patrice Lacombe. Dans ce second cas, il reste à se lier, par l'ultramontanisme comme le montre Monseigneur Bourget, l'évêque de Montréal $^{8}$, et ses agents littéraires comme Jules-Paul Tardivel dans son roman Pour la patrie, à ce qui dépasse

4 Domingo Faustino Sarmiento, Facundo, Barcelona, Planeta, 1975 (1ère ed. 1845).

5 Ottawa, University of Ottawa Press, 2001.

6 Introduction à une poétique du divers, Paris. Gallimard, 2006.

7 Patrick Imbert, Trajectoires culturelles transaméricaines, Ottawa, Presses Université d'Ottawa, 2004, 342 p.

8 Patrick Imbert, « Monseigneur Bourget essayiste », dans L'Essai au Québec, 1985, Collection « Archives des lettres canadiennes », tome VI, p. 319-324. 
le temps long du mythe du progrès : c'est la visée vers l'éternité céleste. Celle-ci est médiatisée par la référence à la Rome universelle catholique et à l'évangélisation. Spatialement, cela se traduit par la mission civilisatrice et catholique des Canadiens français en Oregon et au Chili et la volonté de convertir les païens indigènes dont on essaye parfois de reconnaître la noblesse au 19ème siècle en les comparant aux héros de la Grèce classique ${ }^{9}$ comme le fait Bibaud, artisan d'une esthétique rédemptrice. Ainsi, comme on le voit ici aussi, la durée longue et la manière de la concevoir à toujours à faire avec la question de la reconnaissance ou du rejet de l'altérité et de la manière dont on construit l'autre.

4/ Évidemment, le contrepoint à cette recherche d'une temporalité longue est la rupture. Elle se manifeste aux États-Unis dans la Déclaration d'indépendance et la valorisation de la frontier, espace ouvert et infini, démocratique et favorisant le développement individuel, le tout couplé à la Destinée manifeste ${ }^{10}$, c'est-à-dire à une nouvelle manière d'envisager le vecteur du progrès à partir de la République des États-Unis et de son libéralisme démocratique qui doit être diffusé à la planète entière. Cette frontier spatiale sera vite transformée en frontier industrielle, financière et récemment en frontier de la société des savoirs visant la création infinie de richesses. Dans ce contexte qui se lie désormais aux Amériques entières engagées dans un processus de transformation profond comme on le voit par exemple au Brésil, les dynamiques propres à la « glocalisation», notamment la démocratisation de l'accès et la légitimité des déplacements géographiques, économiques et symboliques, reconfigurent la question de la durée longue dans la rencontre de temporalités et de cultures diverses en processus de transition permanente. Le transculturel ${ }^{11}$, l'interculturel et le multiculturel réorientent dès lors les aspirations à la durée longue et à la stabilité d'une narrativité qui lui serait coextensive.

\section{Les réincarnations : les durées longues, l'éphémère et la rencontre avec l'autre}

celles qui barbouillent leur corps de graffitis à la mine de plomb, gravant leur histoire dans le secret de leur peau, l'éraflant jusqu'au sang, faisant ainsi mentir encre et papier, Herménégilde Chiasson, Béatitudes, Sudbury, Prise de parole, 2007, p. 29.

Comment se manifeste, dans les textes littéraires, la présence de cette transition permanente qui remet en question les légitimations par les grands récits reposant sur une durée longue canonique? Notamment par le jeu sur les réincarnations qui recontextualisent les exclusions générées par le canon historique de la durée longue en rencontres déplaçant le dualisme soi/les autres synonyme de civilisé/barbare et de intérieur/extérieur ${ }^{12}$.

Dans la construction du personnage romanesque constitué non plus comme foyer de cohérence issu du lien entre l'itération des fonctions narratives et des paradigmes sémantiques de la description comme dans le roman réaliste balzacien, mais comme

9 François-Marie Bibaud, Biographie des Sagamos illustres de l'Amérique septentrionale, Montréal, Lovell et Gibson, 1848.

10 Patrick Imbert, « Destino Manifesto » dans Dicionário de Figuras e Mitos Literários das Américas (Zila Bernd, org.), Porto Alegre, Universidade Federal do Rio Grande do Sul et Tomo editorial, 2007, p. 178184.

11 Patrick Imbert, Trajectoires culturelles transaméricaines, Ottawa, Presses de l'Univrsité d'Ottawa. 2004.

12 On pense aussi à Patrick Imbert, Réincarnations, Hull, Vents d'Ouest, 2004 ou encore à Hernán Rivera Letelier, El arte de la resurección, Buenos Aires, Alfaguara, 2010. Dans ce cas, il s'agit d'un illuminé qui pense être Jésus et qui bouscule certaines données établies! 
entité psychologique multiple en transformations majeures issue de traces narratives et contextuelles diverses, les auteurs contemporains des Amériques explorent les scénarios des violences humaines. C'est le cas du montréalais Yann Martel dans Self où le protagoniste est homme puis femme violée, puis homme. Une dynamique similaire s'affiche chez l'écrivaine mexicaine Laura Esquivel dans The Law of Love où la femme autochtone violée par le conquistador (selon le stéréotype narratif traditionnel au Mexique) se réincarne et revient comme homme quelques dizaines d'années plus tard. Elle domine ce conquistador devenu femme. Les réincarnations transsexuelles et transhistoriques transforment les luttes de pouvoir. La déconstruction du personnage romanesque qui révèle des conduites imprévues échappant au canon du vraisemblable qui est inscrit dans l'enchaînement des fonctions narratives reposant sur la coprésence de la temporalité et de la causalité, aboutit à l'exploration ludique des conduites invalidantes.

Surtout, ce que nous disent ces romans remettant en question la cohérence traditionnelle du personnage liée à sa fonction platonicienne mimétique le posant comme transparent, comme représentant d'un discours vraisemblable, est qu'ils ne copient pas le réel. Ils rendent compte des dynamiques propres à la mimésis d'appropriation telle que définie par René Girard dans Des choses cachées depuis la fondation du monde ${ }^{13}$ qui pousse tous contre tous à imiter un modèle indiquant quel est l'objet désirable propre à la quête dans telle ou telle société. Ces romans indiquent d'une part de quelle manière on invente une société et cela en fonction de quel consensus canonique obligeant à oublier quelles victimes et, d'autre part, le potentiel de violence qui se manifeste dans les relations interhumaines ${ }^{14}$.

Ainsi, le corps en processus de réincarnation permet d'explorer les modalités des actes en lesquels le personnage (ou l'institution) ne se reconnaît pas et de le forcer à se reconnaître en eux comme le souligne Kundera. Cette situation notée par Kundera dans l'Art $d u$ roman se manifeste lorsque cet auteur souligne qu'une grande lacune de la civilisation européenne est de ne pas s'être reconnue dans son roman, c'est-à-dire de ne pas vouloir comprendre que les grands romanciers mettent en valeur depuis plusieurs siècles le fait que le personnage romanesque ne se reconnait pas dans ses actes. Cette déconnexion entre des identités qu'on s'attribue et les faits désastreux qui y sont liés est exploitée par Diderot, Sterne, Kafka, Hasek, Havel, etc. On pense en particulier à Kafka dont les romans annoncent la rationalité bureaucratique génocidaire qui se met en place dans l'Allemagne nationaliste sous le nazisme.

De nos jours, cette capacité à percevoir la difficulté à se reconnaître dans ses actes débouche, dans le genre romanesque tel que conçu par Laura Esquivel ou Yann Martel, sur la production de textes qui explorent le positionnement et les souffrances des personnages et de leur corporéité même, par le biais du viol ou de la torture, en fonction des relations de pouvoir, en fonction de l'enjeu même du corps comme réalité biologique située inscrite dans les luttes mimétiques. Pour eux, pour cesser de produire des victimes, il faut être à la place de l'autre, dans son dénuement face aux pouvoirs, par le biais d'un récit noncanonique jouant des réincarnations et qui fait relire les récits canoniques mythiques contrôlés par les lyncheurs. Comme le dit Carlos Monsivais dans son essai intitulé Los

13 Paris, Livre de poche, 1978. Voir Patrick Imbert, Theories of Exclusion and of Inclusion and the KnowledgeBased Society : Canada and the Americas, Ottawa, University of Ottawa Research Chair: "Canada: Social and Cultural Challenges in a Knowledge Based Society" Publisher, 2008, 196 p.

14 Voir aussi Andrew McKenna, Violence and Difference : Girard, Derrida, and Deconstruction. ChampaignUrbana, University of Illinois Press, 1992. 
rituales del caos, commentant les statues qui émaillent les places publiques en Amérique latine et qui manifestent l'euphorie de l'indépendance, elles signifient désormais surtout " Mide tu pequenez frente al Estado! ${ }^{15}$ »

Ces textes de réincarnation et de renaissance explorent la reconnaissance de l'altérité en soi et du soi en l'autre comme l'évoquent Lévinas ${ }^{16}$ et Ricœur ${ }^{17}$. Ils débouchent sur l'ouverture au pluralisme des options en butte à la rigidité de l'orthodoxie fondée sur la durée longue d'un vecteur temporel mythique ou narratif. Les textes littéraires déplacent ainsi la synonymie entre intérieur/extérieur et soi/les autres et créent un espace élargi où les réincarnations ont pour rôle de donner une durée très longue à l'instant où la victime se fait fermer la bouche par les lyncheurs (viol, torture, exécution). En effet, cet instant est à la fois éphémère dans sa violence immédiate et très long par son impact sur la mémoire. Comme l'affirme Girard au sujet de Jésus, « Il faut que cette victime réussisse à nous atteindre au moment où la violence lui ferme la bouche. ${ }^{18} \gg \mathrm{C}$ 'est cet instant crucial qui laisse des traces que Laura Esquivel et Yann Martel jouant d'un certain type de réalisme magique ${ }^{19}$ saisissent et auquel, par les jeux sur les réincarnations ou sur les changements d'orientations sexuelles, ils donnent une ampleur exceptionnelle et originale. Les perspectives plus traditionnelles sur la construction du personnage ne démontent pas le mécanisme aussi efficacement. En effet, si on s'oppose à la violence, c'est par une violence qui permet de triompher, certes, mais c'est une violence contre une violence, qui souvent s'affirme dans sa vérité triomphante. Cet instant éphémère de la violence saisie sur le vif et transformé en durée longue ${ }^{20}$ dans le retentissement de la mémoire opposée à l'histoire officielle, celle de l'histoire nationale diffusée dans les écoles ou celle diffusée sous forme de désinformation dans les médias, met les lecteurs à la place de l'autre, dans sa vulnérabilité face aux pouvoirs.

Il est intéressant de constater que cet instant est transformé en mémoire, en durée teintant d'émotions les diverses périodes de la vie du personnage échappant ainsi en partie à la durée du canon historique que les Amériques recherchent et qui est court par rapport à la durée européenne. La réincarnation est bien un désir d'être autre et d'échapper, en les faisant reconnaître, à des violences insupportables. L'histoire fictionnelle, la narrativité romanesque se sépare donc de l'histoire officielle qui assure ses oublis dans la sélection archivistique reposant sur la durée historique. Les fictions postmodernes/postcoloniales se consacrant à manifester les violences occultées bouleversent à la fois la narrativité vraisemblable et la pseudo-cohérence du personnage traditionnel qui passe par une lente maturation pour être crédible et qui est lié à un territoire qui le définit en rapport avec une durée censée être homogène et partagée par le groupe.

C'est ce lien entre territoire et invention de l'histoire et de ses exclusions qu'explore tout récemment l'auteur québécois Éric Dupont dans La logeuse. On y assiste à la dénégation du meurtre et de l'exclusion qui a lieu à Notre-Dame-du-Cachalot, un petit village de la Gaspésie. Dans celui-ci, les bureaucrates de la pensée dualiste tentent de créer un paradis socialiste et communautaire national protégé du reste du monde à l'époque même

15 Mexico, Era, 1995, p. 148. Nous traduisons : « Mesure ta petitesse face à l’État! ».

16 Emmanuel Lévinas, Totalité et infinité; essai sur l'extériorité, La Haye, Nijhoff, 1961.

17 Paul Ricœur, Oneself as Another, Chicago, Chicago University Press, 1992.

18 Des choses cachées depuis la fondation du monde, Paris, Livre de poche, 1978, p. 317.

19 Pour la présence du réalisme magique dans la littérature québécoise, voir : Catherine Khordoc, «Le diable, l'archange et le génie : Le réalisme magique dans l'œuvre "américaine" de Francine Noël», à paraître.

20 Pour une acception différente de ce concept et son lien avec les identités des Amériques en concurrence avec les identités européennes voir : Gérard Bouchard et Michel Lacombe, Dialogue sur les pays neufs, Montréal, Boréal, 1999. 
de la Pérestroïka et de la déroute des pays socialistes occupés par l'Union soviétique. Ils masquent leur but dominateur tout en organisant une société où l'individu différent n'a pas de place : «Notre-Dame-du-Cachalot, malgré tous les mensonges qui circulent à son sujet, représente l'achèvement le plus méconnu du rêve politique socialiste d'inspiration marxiste ${ }^{21}$. C'est bien pour manifester la désinformation sur laquelle repose ce rêve, ainsi que la dénégation du mensonge, qu'on trouve à l'entrée du village des tumulus pyramidaux dont on dit que ce sont des tombes érigées autrefois par les autochtones. La durée longue précolombienne est donc présente et cautionne les valeurs communautaires et leur pérennité. Toutefois, le narrateur sait à quoi s'en tenir sur la désinformation marxiste. Il nous révèle un rituel plus primaire. À chaque fois qu'un villageois se révèle dans sa singularité, dans sa différence, par exemple par le biais d'un cactus de Noël fleurissant et annonçant les tempêtes hivernales, il est lapidé de manière expéditive par la foule en délire confondant causalité et coïncidence ${ }^{22}$ en croyant que c'est le cactus et le propriétaire du cactus qui causent les tempêtes redoutées. L'individu disparait alors sous un tumulus. Que voilà, de surcroit, une intéressante traduction fictionnelle de la théorie du processus victimaire analysé par René Girard $^{23}$. Que nous voilà loin, dans ce démontage de la logique territoriale unie à l'exclusion, de la capacité de devenir autre manifestée par le personnage principal de La logeuse, Rosa Ost qui s'en va à Montréal, marquée de ces violences et s'insère dans la multiplicité des variations culturelles enserrées toutefois dans le «bordel » d'un capitalisme sauvage: «son monde était un immense bordel dont elle était la tenancière» ${ }^{24}$.

\section{L'Instant, la mémoire et la littérature : le non-relativisme et le rejet du canon}

La littérature, dans sa dynamique de réincarnations donnant accès à l'instant et à ses retentissements dans la mémoire comme on le voit aussi dans la nouvelle « And of Clay are We Created » de Isabel Allende ${ }^{25}$, est bien une fiction complète. Elle est une fiction justement car elle donne voix à l'autre, à celui dont les discours officiels historiques refusent l'existence. Ou plutôt, n'est-ce pas le discours officiel qui est une fiction en ne voulant pas reconnaître la présence de l'autre et lui accorder une place? C'est probablement ce que dirait Oswald de Andrade valorisant la rencontre des cultures autochtones, afro-brésiliennes et européennes si l'on saisit bien ses intentions dans le Manifeste anthropophage. La fiction est le médium qui témoigne du fait qu'un tel est né, a vécu en tel ou tel lieu et a souffert d'exclusion, cela en rappelant la réalité des cadavres banalisés soit sous le spectaculaire des médias soit niés par les régimes répressifs dans la désinformation médiatique et les histoires officielles.

Contrairement à la littérature engagée, celle de Sartre par exemple qui oubliait les dizaines de millions de morts des Goulags, un certain type de fiction, celui qui mêle le baroque, le parodique et le ludique au témoignage, au testimonio, parvient à remettre en question la synonymie soi/les autres, intérieur/extérieur en reconfigurant les étapes des rapports de temporalité et de cause à conséquences fondateurs des oublis de ceux qui partagent un espace et dont les vies ne correspondent pas au vecteur du récit historique

21 Montréal, Marchand de feuilles, 2006, p. 12.

22 Ibid., p. 15.

23 Voilà qui rejoint aussi Vaclav Havel dans Living in Truth. Dans cet essai, il dénonce les horreurs des bureaucraties socialistes où tous participent aux dénonciations menant à la prison, à la torture à des générations complètes aux vies gâchées. Ces régimes ignorent en fait la nécessaire «continuity between peace and human freedom». Vaclav Havel, Living in Truth, London/Boston, Faber and Faber, 1986, p. 188.

24 La logeuse, p. 262.

25 Isabel Allende, «And of Clay Are We Created" dans Stories of Eva Luna, New York, Bantam, 1991. 
canonique privilégié par l'institution. Cette fiction parvient à transformer le territoire restreint, fondement des écritures et des lectures nationales prises dans le jeu à somme nulle des lectures imposées excluant les autres interprétations et notamment la légitimité des discours des victimes, en espaces pluridimensionnels soutenus par la coprésence de récits légitimes non-concomitants. Ils réunissent simultanément en un nouveau métissage culturel ce qui est perçu comme contradictoire dans une logique territoriale et une historicité canonique particulière mais qui ne l'est pas sur le plan de la logique de la société des savoirs. Ces fictions contribuent à déplacer les rapports dualistes toujours contrôlés par un monisme hégémonique et leurs implications gouvernant la manière même dont les rapports économiques et culturels s'inventent.

Le pluralisme littéraire réécrivant les grands récits de légitimation est donc nonrelativiste dans sa reconnaissance qu'il y avait des gens vivants alors que l'arbitraire monologique des institutions les nie souvent. Il est fondé sur l'éthique de la reconnaissance des victimes produites par les discours historiques monistes affirmant détenir la vérité. Il montre les lyncheurs, comme les appelle Girard, ceux qui inventent un discours mythisant ou historique affirmant que la victime était coupable de diviser la communauté ou plus simplement l'oubliant. Dans ce cas, les lyncheurs se présentent comme des sauveurs communautaires rétablissant l'ordre. C'est alors aux textes pluralistes de pointer du doigt le mensonge du lyncheur qui sacrifie les vies au nom de la vérité diffusée par le monopole historico-archivistique, terroriste religieux ou désinformatif-médiatique. Ces textes soulignent l'arbitraire du discours de vérité issu de l'institution et affirment qu'on ne peut dire la vérité car seul Dieu le peut. Par contre, on peut pointer le mensonge. Mentir, dans l'optique de ces textes pluralistes valorisant l'instant violent et son retentissement dans la mémoire, est affirmer détenir la vérité en faisant croire qu'on a accès à un extérieur au discours et qu'il est nécessaire de tuer en son nom pour refonder la cohérence du groupe autour d'un récit homogénéisant naturalisé par un retour aux origines.

\section{Le déjà-là, le multiculturalisme et l'invention des Amériques}

Les cultures ne sont pas des entités autonomes ou statiques. Notre tendance à les considérer comme figées - comme s'il y avait de véritables fractures ou lignes de faille les séparant - constitue l'un des principaux obstacles au dialogue interculturel. (...) Parler de fractures pour décrire les différences entre les cultures revient à ignorer la porosité des frontières culturelles et le potentiel créatif des individus qu'elles abritent. Comme les personnes humaines, les cultures n'existent qu'en relation les unes avec les autres. (UNESCO) ${ }^{26}$.

Toute cette question de la durée longue et des traces mémorielles a un lien avec les rapports socio-économiques quotidiens. En effet, si on ne valorise que le neuf, que la créolisation, comment concevoir aussi que les nouveaux arrivants devraient faire un effort pour se mêler à un déjà-là culturel qui a une valeur certaine, en particulier, ce pourquoi les nouveaux arrivants émigrent, c'est-à-dire un mode de vie libéral et démocratique qui a été inventé petit à petit à partir de Constitutions et de lois qui ont permis le développement de ce mode de vie, ainsi que l'attrait de celui-ci sur les nouveaux arrivants. Simultanément, on doit aussi reconnaître, comme le fait Will Kymlicka, que l'État-Nation même démo-

26 UNESCO, Investir dans la diversité culturelle et le dialogue interculturel. Paris, Rapport mondial de l'UNESCO (résumé), 2009. 
cratique a causé des torts importants à quantité de personnes et de groupes : « Building liberal-democracy on national foundations has historically involved a number of injustices against those who are not seen as full members of the nation, whether they are inside the boundaries of the state or outside $\gg{ }^{27}$. Will Kymlicka, contrairement à Seyla Benhabib qui propose plutôt de construire une citoyenneté cosmopolite ou post-nationale, suggère de domestiquer la violence de l'État-Nation par la mise en place de lois ouvertes sur le multiculturalisme liées à une vision de l'État comme ensemble multinational. Autrement dit, Will Kymlicka propose d'accommoder les immigrants à partir de la reconnaissance d'un déjà-là systémique et culturel, d'une durée longue, celle de la démocratie libérale définie dans le cadre de l'État national. Dans ce contexte de migrations et de reconnaissance des minorités, le philosophe canadien Charles Taylor complémente Kymlicka car il affirme que les valeurs ne sont pas toutes égales. En effet, des cultures établies depuis longtemps ont quelque chose à dire de particulier dans le contexte de l'État-Nation. Même s'il ne précise pas exactement ce que signifie longtemps et en particulier longtemps dans les Amériques, on retient que la distinction d'une durée longue liée à un espace déjà habité est significative et qu'elle a des droits en tant que telle, face à l'hégémonie d'une institution autre ou de manifestations du nouveau par le biais de cultures liées à l'immigration.

Taylor et Will Kymlicka sont, de plus, conscients du fait que le fonctionnement de l'État-Nation ne peut plus être vu comme étant neutre face aux cultures et que le grand récit historique national et censé être homogène diffusé dans la modernité et lié au libéralisme procédural ne peut qu'être repensé. En effet, ce récit national s'est fragmenté en éléments qui reconnaissent l'exclusion des femmes, des gays, des autochtones, des métis et de divers groupes et minorités dont les attributions définissent souvent la personnalité d'un individu participant à plusieurs de ces catégories produisant des narrativités qui sont les mémoires longues des instants violents comme par exemple la pendaison de Louis Riel dans l'Ouest canadien et l'exclusion des métis ou encore comme la marginalisation des francophones d'Edmonton majoritaire dans le nord de l'Alberta avant 1905 mais maintenant très minoritaires ${ }^{28}$.

Dans le contexte où Taylor affirme que les valeurs ne sont pas égales car des cultures établies depuis longtemps, celle du Québec par exemple, ont quelque chose à dire de particulier dans le contexte de l'État-Nation, il est important de savoir de quelle manière on doit voir les autres et comment les rapports peuvent être envisagés en fonction des ressentiments, des violences subies et de l'avenir à envisager ensemble. L'État qui défend, notamment par l'éducation, des fins collectives doit aussi protéger les droits fondamentaux des minorités et des immigrants ce qui est la base du libéralisme substantif qui ne doit pas non plus oublier les droits individuels. Voilà qui évite le relativisme absolu pour déboucher sur une conception de la culture comme relations multiples en évolution toujours fondées sur des expériences et des contextes déjà établis mais qui sont en transition permanente dans leurs relations à d'autres cultures et à d'autres systèmes de valeurs se déplaçant aux rythmes des rencontres et de la glocalisation ainsi que le soulignent Frederick Barth ${ }^{29}$ ou Amartya $\mathrm{Sen}^{30}$.

27 Will Kymlicka, « Liberal Nationalism and Cosmopolitan Justice” dans Seyla Benhabib (ed. Robert Post), Another Cosmopolitanism, Oxford, Oxford University Press, 2006, p. 129.

28 Alberta, village sans mur (s), dir. Estelle Dansereau, Paul Dubé, Eileen Lohka, Pamela V. Sing, Winnipeg, Presses universitaires de Saint-Boniface, 2005.

29 Frederick Barth, «Les groupes ethniques et leurs frontières », Théorie de l'ethnicité, Presses universitaires de France, Paris, 1995, pp. 203-249.

30 Identité et violence, Paris, Odile Jacob, 2001. 
Ainsi, la durée longue dans la pratique de la reconnaissance et de la gestion de la relation, rejoint des considérations non seulement artistiques mais aussi socio-économiques et légales. En effet, un État libéral peut défendre non pas une seule fin collective mais des fins collectives qui reposent sur la promotion de certains droits particuliers réclamés par certaines minorités qui ne réclament pas toute la même chose : droits de chasse pour certains groupes autochtones, droits linguistiques pour d'autres groupes, etc. Il y a donc continuité d'une moralité politique appliquée par les juges, le pouvoir exécutif, les pédagogues, etc., qui doit tenir compte de la finalité collective d'une communauté grâce à laquelle se développent un individu et des groupes. Un exemple récent est la décision de contester avec succès la décision du gouvernement conservateur de Mike Harris en Ontario de fermer l'Hôpital francophone et bilingue universitaire Montfort d'Ottawa pour des raisons économiques. Il s'est alors s'agit, en plus de mobiliser la population, de démontrer qu'il y avait discrimination contre un groupe, les Francophones minoritaires de l'Ontario, et pour cela il fallait reconnaître que l'hôpital Montfort à Ottawa constitue aussi une entité culturelle et sociale pour les francophones de l'Ontario qui ont droit a être soignés dans les meilleures conditions de compréhension culturelle et linguistique car la santé des populations est aussi une question de rapport, d'écoute et de saisie des maux qui ne sont pas uniquement définis par des bactéries ou des virus. Pour le Québec cela signifie de s'engager dans l'interculturalisme qui demande aux communautés culturelles d'envoyer leurs enfants dans les écoles francophones en fonction de la loi 101 et des perspectives marquées par la dynamique des accommodements raisonnables : «L'interculturalisme s'efforce de concilier la diversité ethnoculturelle avec la continuité du noyau francophone (...), il a la vertu d'être flexible, ouvert à la négociation, aux adaptations et aux innovations $»^{31}$. Ainsi, les traces se glissent dans le déjà-là d'une continuité fortement soulignée.

Les lois, les théories multiculturelles et les productions artistiques et littéraires convergent ainsi vers une réflexion sur les pratiques relationnelles permettant d'inventer des rapports socio-économico-culturels plus harmonieux qui permettront à toutes et à tous de travailler ensemble à s'épanouir en fonction d'une promesse d'avenir meilleur. Cet avenir meilleur est ce qui donne aussi une durée longue, quoiqu'imprévisible, aux fragments, aux traces, aux vestiges qui se perpétuent dans les instants et les temps forts des rencontres fondées sur l'exclusion comme sur la séduction menant à la créolisation. C'est ce que rappelle Nicolas Dickner dans Nikolski en présentant l'épopée des Garifunas ni tout à fait amérindiens ni tout à fait africains éparpillés suite aux traités signés par les puissances coloniales françaises et anglaises, le long des côtes du Nicaragua, au Honduras et au Belize : «Et personne, pas même les plus grands ethnologues, ne comprend bien le subtil mécanisme grâce auquel ces orphelins ont pu, malgré les déracinements et l'exil, préserver leur identité $\aleph^{32}$. La créolisation séduit de plus en plus dans la civilisation contemporaine inventée sur la légitimation des déplacements géo-symboliques. C'est pourquoi Édouard Glissant nous rappelle qu'il faut étudier constamment le retentissement de « ce

31 Fonder l'avenir, le temps de la conciliation : Commission de consultation sur les pratiques d'accommodement reliées aux différences culturelles, Rapport par Gérard Bouchard et Charles Taylor, Saint-Lazare, Québec, 2008, p. 21.

32 Québec, Alto, 2007, p. 225. On retient toutefois que l'éditeur qui publie des auteurs québécois et des auteurs en traduction qui ne sont pas du Québec affiche ce qui suit sur son site web : « Nous n'acceptons pas pour l'instant de soumissions d'auteurs francophones habitant hors Québec ». (02/10/2010). Le canonique de l'exclusion des francophones hors Québec est affiché par l'éditeur. 


\section{Conexão Letras}

qui change en s'changeant » dans des Amériques qui « s'archipélisent » de plus en plus dans $«$ le besoin que les cultures ont de toutes les cultures $»^{33}$.

\section{Conclusion}

De la durée longue et du monologique on aboutit au transculturel ${ }^{34}$ qui joue des relations créant du nouveau ou le continu tend à se tracer dans la trace et le discontinu. C'est celui-ci qui est exploré par quantité d'auteurs et d'artistes où le caméléonage est de s'adapter au contexte comme le souligne Dany Laferrière : «'Êtes-vous un écrivain haïtien, caribéen ou francophone?' Je répondis que je prenais la nationalité de mon lecteur. Ce qui veut dire que quand un japonais me lit, je deviens immédiatement un écrivain japonais $»^{35}$. Mais c'est aussi savoir que le contexte est lui-même bigarré, hétérogène et que plus que de s'adapter, il s'agit d'être efficace dans le multiple et le chatoyant du changement ce que souligne le peintre Cajun George Rodrigue au sujet de son chien bleu : The wonderful thing about Blue Dog is that she's never hung up on playing a specific role; she will happily inhabit any world I put her in. Within Blue Dog is the history of the Cajun people and the history of myself, my joys and fears and visions, both of the world around me and the world yet to come... Through Blue Dog I can process the world around me, with all its complexity. Color, noise, and absurdity. The paintings in this book reflect this new path ${ }^{36}$.

Voila qui rejoint des dynamiques de la vie quotidienne telle que les souhaite Piscine Patel dans L'Histoire de Pi de Yann Martel où, intéressé par la métaphysique plus que par le communautaire, il veut à la fois vivre le bouddhisme, l'islam et le christianisme. Ces dynamiques des croisements qui laissent des traces sont aussi exprimées par les membres d'une famille mixte à Toronto: "Even though most of his family members are Muslim, Boudjenane says that 'because his sister-in-law and niece are Christians, the whole family celebrates both Christmas and Ramadan. That's what being Canadian is all about", he says" ${ }^{37}$. La société des savoirs glocalisée mène donc à produire des significations diverses liées à la réflexivité et à des savoirs sur les processus, les codes et les dynamiques de pouvoir où on s'invente non comme identité stable mais avec diverses images de soi permettant d'être producteur d'avantages différentiels et séducteurs dans la collaboration.

\section{Referências}

ALLENDE, Isabel. And of Clay Are We Created. In: Stories of Eva Luna. New York: Bantam, 1991.

33 Introduction à une poétique du divers, Paris, Gallimard, 1996, p. 32, 44 et 132.

34 Afef Benessaieh, 'Amériques transculturelles?' dans A. Benessaieh (dir.) Transcultural Americas/Amériques transculturelles, Ottawa, Presses de l'Université d'Ottawa, p. 1-10.

35 Dany Laferrière, Je suis un écrivain japonais, Montréal, Boréal, 2008, p. 30.

36 George Rodrigue and Tom Brokaw, Blue Dog Man, New York, Stewart, Tabori and Chang, 1999, p. 21. Nous traduisons : « La chose merveilleuse à propos de Blue Dog est qu'elle ne se raccroche jamais à un rôle spécifique, elle habitera quelque monde dans lequel je l'insèrerai. Au sein de Blue Dog réside l'histoire du peuple Cajun et mon histoire, mes joies, mes peurs et mes visions, à la fois le monde autour de moi et le monde à venir...A travers Blue Dog je peux traiter le monde autour de moi, avec toute sa complexité. Couleur, bruit, et absurdité. Les peintures dans ce livre reflètent ce nouveau chemin ».

37 Joan Delaney, "Political correctness gone too far?", The Epoch Times, December 22-28, 2006, p. 1. Nous traduisons: Quoique la plupart des membres de sa famille soient musulmans, Boudjenane dit que' étant donné que sa belle-soeur et sa nièce sont chrétiennes, toute la famille fête à la fois Noël et le Ramadan.. Voilà ce que c'est que d'être Canadien", dit-il". 
BARTH, Frederick. Les groupes ethniques et leurs frontières. In: Théorie de l'ethnicité. Paris: Presses universitaires de France, 1995.

BENESSAIEH, Afef. ‘Amériques transculturelles?' In: BENESSAIEH, A. (org)

Transcultural Americas/Amériques transculturelles. Ottawa: Presses de 1'Université d'Ottawa, 2010.

BIBAUD, François-Marie. Biographie des Sagamos illustres de l'Amérique septentrionale. Montréal: Lovell et Gibson, 1848.

BOUCHARD, Gérard. Genèse des nations et cultures du Nouveau Monde. Montréal: Boréal, 2001.

. TAYLOR, Charles. Fonder l'avenir, le temps de la conciliation: Commission de consultation sur les pratiques d'accommodement reliées aux différences culturelles.

Saint-Lazare: Québec, 2008.

CHEN, Ying. Quatre mille marches. Montréal: Boréal, 2004.

DELANEY, Joan. "Political correctness gone too far?" The Epoch Times, December 22-28, 2006.

DICKNER, Nicolas. Nikolski. Québec: Alto, 2007.

DUPONT, Éric. La logeuse. Montréal: Marchand de feuilles,. 2006.

GIRARD, René. Des choses cachées depuis la fondation du monde. Paris: Livre de poche, 1978.

GLISSANT, Édouard. Introduction à une poétique du divers. Paris: Gallimard, 1996. HIGHWAY, Tomson. Comparing Mythologies. Ottawa: University of Ottawa Press, 2001. IMBERT, Patrick. Monseigneur Bourget essayiste. In: L'Essai au Québec. Collection " Archives des lettres canadiennes », tome VI, 1985.

. Trajectoires culturelles transaméricaines. Ottawa: Presses Université d'Ottawa, 2004.

. Destino Manifesto. In: BERND, Zilá (org). Dicionário de Figuras e Mitos Lite-

rários das Américas. Porto Alegre: Universidade Federal do Rio Grande do Sul e Tomo editorial, 2007.

KYMLICKA, Will. Liberal Nationalism and Cosmopolitan Justice. In: BENHABIB, Seyla. (ed. Robert Post). Another Cosmopolitanism. Oxford: Oxford University Press, 2006.

LAFERRIÈRE, Dany. Je suis un écrivain japonais, Montréal: Boréal, 2008.

LÉVINAS, Emmanuel. Totalité et infinité; essai sur l'extériorité. La Haye: Nijhoff, 1961.

MONSIVAIS, Carlos. Los rituales del caos. Mexico: Era, 1995.

RICCEUR, Paul. Oneself as Another. Chicago: Chicago University Press, 1992.

RODRIGUE, George; BROKAW, Tom. Blue Dog Man, New York: Stewart, Tabori and Chang, 1999.

SEN, Amartya. Identité et violence. Paris: Odile Jacob, 2001.

SARMIENTO, Domingo Faustino. Facundo. Barcelona: Planeta, 1975 (1ère ed. 1845). UNESCO, Investir dans la diversité culturelle et le dialogue interculturel. Paris: Rapport mondial de l'UNESCO (résumé), 2009. 